\title{
PLASMA LUMINESCENCE WITH WATER DROPLETS AND VAPOR
}

\author{
N. Kondo, H. Ofuruton* and Y. H. Ohtsuki \\ Department of Physics, Waseda University \\ 4-1, Okubo 3, Shinjuku-ku, Tokyo 169, Japan \\ *Tokyo Metropolitan College of Aeronautical Engineering \\ 52-1, Minamisenju 8, Arakawa-ku, Tokyo 116, Japan
}

\begin{abstract}
A lot of meteorologists and physicists have accepted ball lightning phenomena to exist as a kind of meteorological phenomena. Ohtsuki and Ofuruton(1991) succeeded in producing plasma luminous objects in the natural atmosphere by microwave interference and showed that ball lightning was a plasma state of the natural atmosphere. A lot of eyewitnesses reported ball lightning in situations with high humidity such as rainy weather, vicinity of lakes or marshes. In this paper, we report an experiment to produce plasma luminous objects by microwave interference in a high humidity atmosphere where water droplets and vapor existed. We confirmed that the produced plasma luminous objects grew and changed the color into red in comparison with the case that the water droplets and vapor do not exist. There are various size and colors of ball lightning in nature. We showed that the reason is an effect of molecules included in the plasma luminous objects.
\end{abstract}

\section{Introduction}

A lot of eyewitnesses have reported spherical luminous objects in weather condition such as thunderstorms(Singer ,1977; Barry, 1980; Egely, 1988; Smirnov, 1993). People call these luminous objects ball lightning. The typical diameter of ball lightning is about $20 \mathrm{~cm}$ and the typical lifetime is about 10 to 20 seconds. A lot of ball lightnings are warm color (red, orange, etc.) and cool-colored (blue, green, etc.) ball lightning is sometimes observed(Egely, 1988). A lot of scientific researchers have collected and analyzed the information about ball lightning and investigated them theoretically and experimentally up to now(Ohtsuki(ed.), 1988; Smirnov, 1993). Nevertheless, we, the scientists, have not obtained a definite scientific answer yet. Because ball lightning is a rare phenomenon, some scientists had thought that ball lighting had been optical illusions or psychological hallucination. However, a lot of physicists and meteorologists have accepted ball lightning as one of physical phenomena recently because there are many pieces of observation information which are anti-examples against illusions and hallucination.

Kapitza(1955) submitted a model of ball lightning which was formed by standing waves of high frequency radio waves. According to his theory, electromagnetic waves produced by torpedoes are reflected on the ground and strong standing waves are made between the clouds and the ground. And then the air is in a plasma state at the antinode of the standing waves because the electromagnetic field is strong there, and the plasma emits light.

Sall'(1992) made a model of ball lightning in which many droplets exist. In his model, the light emits by the streamer corona. He discussed the color of ball lightning. His main results were following: ball lightning became red by increasing the diameter, the intensity of the light became strong by increasing the density of the droplets, and blue ball lightnings were seen in the fine weather.

In 1991, our research group carried out an experiment based on Kapitza's theory. 
And we were successful in producing plasma luminous objects which were the natural atmosphere in a plasma state by microwave interference in a cavity at 1 atm and reproducing ball lightning phenomena experimentally(Ohtsuki and Ofuruton, 1991; 1994). The characters of the plasma luminous objects (ball lightning in a laboratory system) are as follows.

(1) Size: about $2-3 \mathrm{~cm}$ in diameter

(2) Shape: flame-like

(3) Color: blue, bluish white (sometimes red, orange, etc.)

(4) The plasma luminous objects occasionally move windward against the wind pressure when the wind is made in the cavity artificially. This means that the movement of the plasma luminous objects is ruled by the electromagnetic wave mode because the electromagnetic wave mode is unrelated to the flow of atmosphere.

Causes of ball lightning formation are radio waves produced by lightning discharges according to Kapitza's theory. However, actual ball lightning is witnessed in weather conditions which are not only thunderstorm but also rain. Besides, many eyewitnesses have reported ball lightning near lakes or marshes, and ball lightning produced under water and rising toward the water surface(Egely, 1998). There are a lot of ball lightning in the places with a large amount of water and vapor as stated above, so that we have to take influences of water on ball lightning formation into account. For this reason, we carried out an experiment of producing plasma luminous objects by the microwave interference under the condition with the water droplets and vapor as well as the eyewitness reports and we examined characteristics of the plasma luminous objects under the condition. In this paper, we mention the experiment, the characteristics of the plasma luminous objects and some discussions about relations between ball lightning and water.

\section{Experiment}

A schematic diagram of the apparatus of our experiment is shown in Fig.1. A magnetron which we used was able to generate $2.45 \mathrm{GHz}, 5 \mathrm{~kW}$ (maximum power) continuous microwaves. The magnetron was connected with a cylindrical cavity $(161 \mathrm{~mm}$ in diameter, $370 \mathrm{~mm}$ in length) by a rectangle waveguide $(55 \mathrm{~mm} \times 109 \mathrm{~mm})$. The waveguide was connected to the cylindrical cavity in such a way that the long side of the waveguide was parallel to the center axis of the cavity. The cavity was made of punched aluminum plates to observe plasma emission in the cavity. A tube $(2 \mathrm{~cm}$ in diameter) was connected with the other round bottom of the cavity at the center. A water current went into the cavity through the tube. The water current became innumerable water droplets by covering the exit of the water current with a punched aluminum plate and the water droplets were scattered in the cavity. The cavity contained the natural air (room temperature and pressure) and the water droplets. The surroundings of the cavity were the natural atmosphere. Plasma luminous objects were produced by the microwave interference in the cavity. We recorded the appearance of the objects with a video camera.

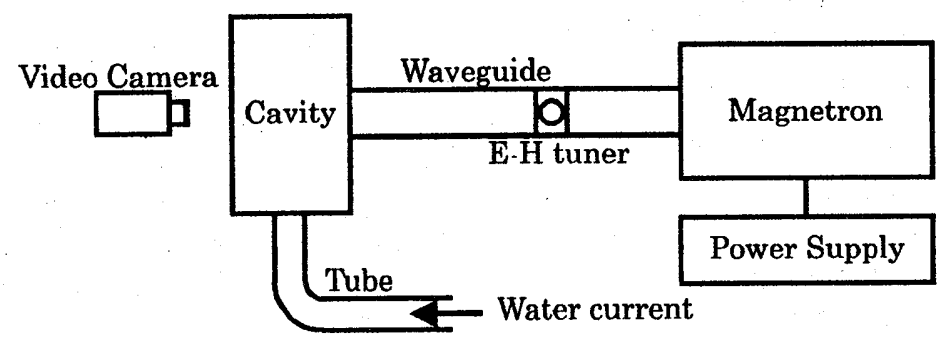

Fig.1 Schematic diagram of the apparatus. 


\section{Results and Discussion}

When the magnetron was turned on without jetting the water droplets, a blue plasma luminous object was produced as shown in Fig.2.

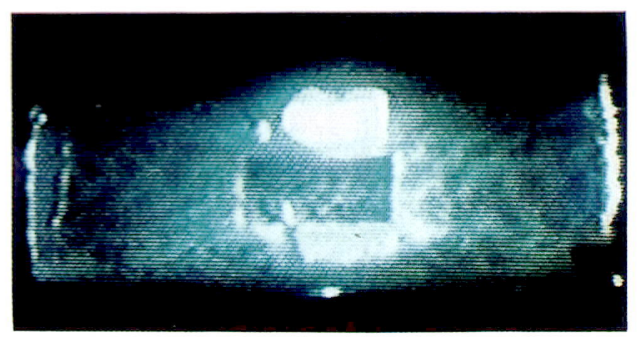

Fig. 2 A typical plasma luminous object in the natural atmosphere.

The jet of the water droplets was started after that, and then the water droplets in the cavity became vapor due to dielectric heating. The water droplets and vapor filled the cavity. Figure 3 shows the appearances of the plasma luminous objects accompanied by the water droplets and vapor. About $5 \mathrm{~kW}$ in the power of the microwave are necessary for continuous existence of the plasma luminous object in case that the plasma object is accompanied by the water droplets and vapor though $3 \mathrm{~kW}$ are enough in case that the plasma object is in the natural atmosphere without water droplets and vapor. Movement of the plasma luminous objects is represented in three photographs in Fig. 3 . The water droplets flowed from the right side to the left side of Fig.3. One plasma luminous object floated in the vicinity of the center of the cavity (Fig.3(a)). The object divided into two pieces, and one of the divided objects went to the right against the water current (Fig.3(b)). And the objects were united with one piece again at the right side in the cavity (Fig.3(c)). Time from Fig.3(a) to Fig.3(c) was about 1 second.

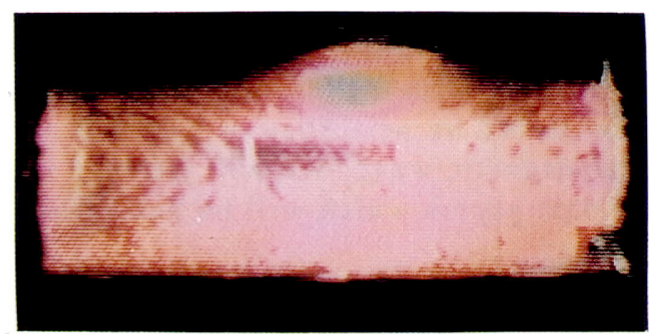

(a) One object was floating in the cavity.

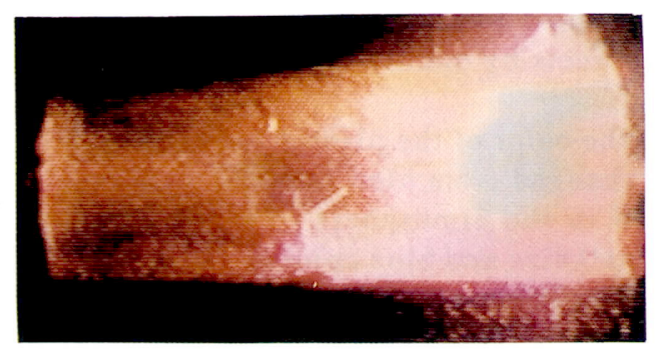

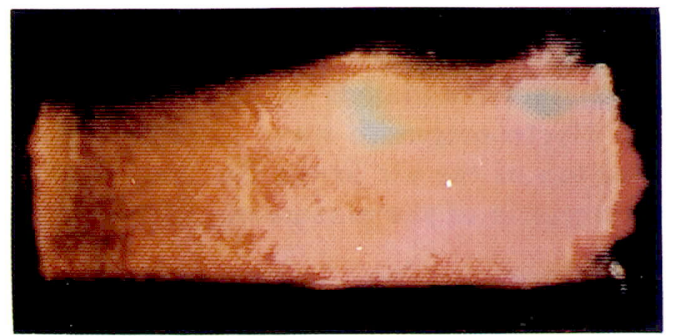

(b) The object was dividing into two pieces.

(c) The two objects were united with one piece again.

Fig. 3 Movement of plasma luminous objects with water droplets and vapor. 
There were some differences of characteristics of plasma objects between with and without water. In the former case, the size of the object was about $2-3 \mathrm{~cm}$ and the color was blue. On the other hand, in the latter case, we could not know the size of the plasma object exactly because the luminous area spread wide in the cavity and the shape of the object was indefinite.

The spread luminescence area means that a larger amount of air is in a plasma state. Even so, the reason has not been clear yet. It is likely that the characteristic is relevant to a difference between the ionization cross-section of the water molecule and that of nitrogen and oxygen which are principal ingredients of the natural atmosphere.

The luminescent color of the plasma with water was red. But we do not know the source of the luminescence because any spectroscopic analyses were not done. However, it is known that plasma luminescence is caused by acceleration motion of charged particles (bremsstrahlung), deexcitation of excited electrons and recombination of charged particles in plasma. In case of the deexcitation and the recombination, the luminescent colors are closely connected with the kind of molecules in the plasma. In our experiment, a large amount of water molecules must have been in a plasma state. Thus, it is thought that water molecules included in the plasma changed the luminescent color into red. This experimental result suggests that humidity of the atmosphere is low if color of ball lightning observed in nature is blue and the humidity is high if the color is red.

These two types of our experimental plasma objects had not only different characteristics but also a common characteristic. The plasma object with water moved against the water current occasionally. The movement was similar that the plasma object without water moved against the flow of air. The characteristic was also evidence that the plasma luminous objects were ruled by not the media but the electromagnetic wave mode. The refractive index of the plasma objects was larger than that of the surrounding natural atmosphere because of the large electric conductivity of the plasma, so that the wave length of the electromagnetic wave which penetrated into the plasma objects became short, and then the energy of the electromagnetic wave concentrated in the objects. Therefore, the intensity of the electromagnetic wave was always strong inside the plasma objects. We consider that the plasma objects continued to move together with the high intensity region of the electromagnetic wave after they started to be moved by perturbation which is the convection of the air or the fluctuation of the electromagnetic wave. We will mention the characteristic of the plasma objects in detail in our next papers which will be published.

\section{Conclusions}

There are a lot of eyewitness reports of ball lightning in rain or in high humidity places. Taking the eyewitness reports into consideration, we carried out an experiment to examine the influence of water droplets and vapor on ball lightning formations. And, we confirmed to be able to produce plasma luminous objects by microwave interference in the atmosphere with water droplets and vapor.

We confirmed the change in the size and the color of the plasma luminous objects also. We showed that the size grew and the color changed from blue into red when a large amount of water droplets and vapor was included in the atmosphere. The result of our experiment means that ball lightning in nature is small size and blue color in the dry air and ball lightning is large and red in the humid air. These results correspond with the theoretical results in which the blue ball lightning is seen in the fine weather and the color of the large size ball lightning is red(Sall', 1992). It was clarified that those characteristics of ball lightning depend on the kind of molecules in the air which are in a plasma state. Therefore, various sizes and colors of ball lightning have been observed in nature. 
Our experimental plasma objects with and without water had a common characteristic. The plasma objects without water moved against the flow of atmosphere and the plasma object with water moved against the water current. We consider that the characteristic is the evidence that the plasma luminous objects are ruled by not the media but the electromagnetic wave mode.

Acknowledgment. The authors would like to thank Mr. H. Nonaka, Mr. M. Kamogawa and the members of Ohtsuki laboratory for their useful discussions and their help.

\section{References}

Barry, J. D., Ball Lightning and Bead Lightning, Plenum Press, New York, 1980. Egely, G., The Mysterious Ball Lightning, Muszaki Konyvkiado, Budapest, 1988.

Kapitza, P. L. , On the nature of ball-lightning, Dokl. Akad. Nauk SSSR, 101, 245-248, 1955(in Russian) (English translation: Collected Papers of Kapitza, Vol.2, ed. By D. Ter Haar, Pergamon Press, London, 776-780, 1965).

Ohtsuki, Y. H. ed., Science of Ball Lightning(Fire Ball), World Scientific Publishing, Singapore, 1988.

Ohtsuki, Y. H., and H. Ofuruton, Plasma fireballs formed by microwave interference in air, Nature, 350, 139-141, 1991.

Ohtsuki, Y. H. and H. Ofuruton, Nature of fireballs produced by microwave interference, Dusty and Dirty Plasmas, Noise, and Chaos in Space and in the Laboratory ed.

H. Kikuchi, Plenum Press, New York, pp.577-579, 1994.

Sall', S.A., On the nature of radiation emission from ball lightning, Sov. Phys. Tech. Phys., 37, 685-688, 1992.

Singer, S., The Nature of Ball Lightning, Plenum Press, New York, 1971.

Singer, S., in: Lightning ed. R. H. Golde, Academic Press, London pp.409, 1977.

Smirnov, B.M., Physics of ball lightning, Physics Reports, 224, 151-236, 1993.

(Received May 14, 1997; revised July 24, 1997; accepted July 28, 1997.) 